\title{
ON THE VARIATIONAL STABILITY OF A CLASS OF NONLINEAR PARABOLIC OPTIMAL CONTROL PROBLEMS
}

\author{
NIKOLAOS S. PAPAGEORGIOU ${ }^{1}$
}

(Received 15 March 1995; revised 16 November 1995)

\begin{abstract}
In this paper we study parametric optimal control problems governed by a nonlinear parabolic equation in divergence form. The parameter appears in all the data of the problem, including the partial differential operator. Using as tools the $G$-convergence of operators and the $\Gamma$-convergence of functionals, we show that the set-valued map of optimal pairs is upper semicontinuous with respect to the parameter and the optimal value function responds continuously to changes of the parameter. Finally in the case of semilinear systems we show that our framework can also incorporate systems with weakly convergent coefficients.
\end{abstract}

\section{Introduction}

In this paper we study the dependence of a nonlinear optimal control problem on a parameter. The parameter appears in all the data of the problem, including the partial differential operator. First we establish the nonemptiness of the set of optimal "state-control" pairs and then we investigate how this set as well as the value of the problem respond to changes of the parameter. Such sensitivity analysis (also known in the literature as "variational stability"), is important because it gives us information concerning the tolerances that are permitted in the specification of mathematical models, it suggests ways to solve parametric problems and can also give us valuable insight for the computational treatment of the problem.

Our tools are the $G$-convergence of operators and the $\Gamma$-convergence of functionals. Using these two convergence concepts, we derive continuous dependence results. Our approach follows that of Buttazzo-Dal Maso [2], who examined linear elliptic control systems and systems monitored by ordinary differential equations. Here we consider parabolic systems with nonlinear dynamics.

$\Gamma$-convergence is a convergence notion of sequences of functions specially designed

\footnotetext{
'National Technical University, Department of Mathematics, Zografou Campus, Athens 15780, GREECE (C) Australian Mathematical Society, 1997, Serial-fee code 0334-2700/97
} 
in order to study convergence of solutions and values of corresponding minimization problems; that is, is a "variational convergence". Among variational convergences, $\Gamma$-convergence plays an important role for its nice compactness properties and for the powerful results it generates concerning the limits of integral functionals. In addition, $\Gamma$-convergence is closely related to the notion of $G$-convergence, which is used in the study of the convergence properties of the solutions of a sequence of elliptic and parabolic problems. Finally, almost all other variational convergences can be easily expressed in the language of $\Gamma$-convergence. Hence it seems appropriate to use these notions to study the variational stability (sensitivity) of optimal control problems.

\section{Preliminaries}

Let $H$ be a separable Hilbert space of norm $|\cdot|$. Let $X$ be a reflexive, separable Banach space with dual $X^{*}$ such that $X \subseteq H \subseteq X^{*}$ with dense and compact injections. The norms of $X$ and $X^{*}$ will be denoted by $\|\cdot\|$ and $\|\cdot\|_{*}$ respectively. We will use $\left\langle x, x^{*}\right\rangle$ to denote the duality brackets between $x \in X$ and $x^{*} \in X^{*}$. This coincides with the inner product in $H$, whenever $x^{*} \in H$. Such a triple of spaces is usually known in the literature as "evolution triple" (see Zeidler [13]; the names "Gelfand triple" or "spaces in normal position" are also used). In concrete applications, evolution triples are generated by Sobolev spaces (see Section 3 ). Let $T=[0, b]$ and define $W(T)=\left\{x \in L^{2}(T, X): \dot{x} \in L^{2}\left(T, X^{*}\right)\right\}$. In this definition, the derivative of $x$ is understood in the sense of vector-valued distributions. Furnished with the norm $\|x\|_{W(T)}=\left[\|x\|_{L^{2}(T, X)}^{2}+\|\dot{x}\|_{L^{2}\left(T, X^{*}\right)}^{2}\right]^{1 / 2}, W(T)$ becomes a Banach space which is separable and reflexive. Furthermore $W(T)$ embeds continuously in $C(T, H)$ and compactly in $L^{2}(T, H)$. When $X$ is a Hilbert space too, then so is $W(T)$ with inner product $(x, y)_{W(T)}=(x, y)_{L^{2}(T, X)}+(\dot{x}, \dot{y})_{L^{2}\left(T, X^{*}\right)}$. For further details we refer Zeidler [13] (Proposition 23.23, pp. 422-423 and p. 450).

Following Kolpakov [6], we say that a sequence of operators $A_{n}: X \rightarrow X^{*} G$ converges to an operator $A: X \rightarrow X^{*}$, if for all $n \geq 1, A_{n}^{-1}, A^{-1}: X^{*} \rightarrow X$ are defined and for every $x^{*} \in X^{*}, A_{n}^{-1} x^{*} \stackrel{w}{\rightarrow} A^{-1} x^{*}$ in $X$ (hence strongly in $H$ ). We will use the symbol $G$ to indicate $G$-convergence. This is a nonlinear version of a convergence concept introduced first by Spagnolo [11] for linear parabolic and elliptic equations and was later extended to abstract linear evolution equations by Zhikov-Kozlov-Oleinik [14].

Next, following Buttazzo-Dal Maso [2] and Buttazzo [1] (Chapter 5), we introduce the notion of multiple sequential $\Gamma$-convergence. So let $X_{1}, X_{2}$ be two topological spaces and let $f_{n}: X_{1} \times X_{2} \rightarrow \overline{\mathbb{R}}=\mathbb{R} \cup\{-\infty,+\infty\} n \geq 1$ be a sequence of functionals. We indicate by $Z(+)$ the sup operator and by $Z(-)$ the inf operator, Let $\left(x_{1}, x_{2}\right) \in X_{1} \times X_{2}$ and denote by $S_{i}, i=1,2$, the set of all sequences in $X_{i}$, 
converging to $x_{i}$. Finally let $\alpha_{j}, j=0,1,2$ be one of the signs + or - . We define

$$
\begin{aligned}
& \Gamma_{\text {seq }}\left(\mathbb{N}^{\alpha_{0}}, X_{1}^{\alpha_{1}}, X_{2}^{\alpha_{2}}\right) f_{n}\left(x_{1}, x_{2}\right) \\
& =\underset{\left(x_{1}^{n}\right) \in S_{1}}{Z\left(\alpha_{1}\right)} \underset{\left(x_{2}^{n}\right) \in S_{2}}{Z\left(\alpha_{2}\right)} \underset{k \in \mathbb{N}}{Z\left(-\alpha_{0}\right)} \underset{n \geq k}{\left(\alpha_{0}\right)} f_{n}\left(x_{1}^{n}, x_{2}^{n}\right) .
\end{aligned}
$$

So for example $\Gamma_{\text {seq }}\left(\mathbb{N}^{+}, X_{1}^{-}, X_{2}^{+}\right) f_{n}\left(x_{1}, x_{2}\right)=\inf _{x_{1}^{n} \rightarrow x_{1}} \sup _{x_{2}^{n} \rightarrow x_{2}} \varlimsup_{n \rightarrow \infty} f_{n}\left(x_{1}^{n}, x_{2}^{n}\right)$.

When the $\Gamma_{\text {seq }}$-limit is independent of the sign + or - , associated with one of the spaces, then this sign is omitted. So for example, if

$$
\Gamma_{\text {seq }}\left(\mathbb{N}^{+}, X_{1}^{-}, X_{2}^{+}\right) f_{n}\left(x_{1}, x_{2}\right)=\Gamma_{\text {seq }}\left(\mathbb{N}^{-}, X_{1}^{-}, X_{2}^{+}\right) f_{n}\left(x_{1}, x_{2}\right)
$$

then their common value will be indicated by

$$
\Gamma_{\mathrm{seq}}\left(\mathbb{N}, X_{1}^{-}, X_{2}^{+}\right) f_{n}\left(x_{1}, x_{2}\right) \text {. }
$$

If the topological spaces are first countable, then the above definition is equivalent to the original topological definition of the $\Gamma$-limits (see Dal Maso [3], Proposition 8.1, p. 86). This is also the case in Banach spaces with a separable dual, equipped with the weak topology and in reflexive Banach spaces again with the weak topology (see Dal Maso [3], Chapter 8). The theory of $\Gamma$-convergence is an important tool in Optimal Control and in the Calculus of Variations, because the equicoercivity and the $\Gamma$-convergence of a sequence of functionals $f_{n}$ to $f, f$ not identically $+\infty$, imply the convergence of the minimizers $\left(x_{n} \rightarrow x\right)$ and of the corresponding minimal values $\left(f_{n}\left(x_{n}\right) \rightarrow f(x)\right)$ (see Theorem 7.19, p. 80 of Dal Maso [3]). The interested reader can find a comprehensive introduction to the subject of $\Gamma$-convergence and its applications, in the well-written monographs of Buttazzo [1] and Dal Maso [3].

Next we introduce our optimal control problem. So let $T=[0, r]$ and $Z$ a bounded domain in $\mathbb{R}^{N}$ with smooth boundary $\Gamma=\partial Z$. Let $/ \Lambda$ be a complete metric space of $\mathbb{R}^{k}$-valued, measurable functions defined on $Z$ (the parameter space) with metric $d(\cdot, \cdot)$. We will be studying the following optimal control problem, parametrized by elements in $\Lambda$ :

$$
\left\{\begin{array}{c}
\int_{0}^{r} \int_{z} L(t, z, x(t, z), u(t, z), \lambda(z)) d z d t \rightarrow \inf =m(\lambda) \\
\text { s.t. } \frac{\partial x}{\partial t}-\operatorname{div}(a(z, D x(t, z), \lambda(z))=f(t, z, x(t, z), \lambda(z)) u(t, z) \quad \text { a.e. } \\
\left.x\right|_{T \times \Gamma}=0, x(0, z)=x_{0}(z), x_{0}(\cdot) \in L^{2}(Z) \\
|u(t, z)| \leq \theta(t, z, \lambda(z)) \text { a.e. with } u(\cdot, \cdot) \text { measurable. }
\end{array}\right\}
$$

Here $D x=\left(D_{1} x, \ldots, D_{N} x_{N}\right)$, with $D_{k}=\frac{\partial}{\partial z_{k}} k=1, \ldots, N$, denotes the gradient of $x$. Throughout this paper the following hypotheses will be in effect. 
$H(a): a(z, v, \lambda)=D_{v} \varphi(z, v, \lambda)$, where $D_{v}$ denotes gradient with respect to the $v \in \mathbb{R}^{N}$ variable and $\varphi: Z \times \mathbb{R}^{N} \times \mathbb{R}^{k} \rightarrow \mathbb{R}$ is a function satisfying:

(1) $(z, \lambda) \rightarrow \varphi(z, v, \lambda)$ is measurable,

(2) $v \rightarrow \varphi(z, v, \lambda)$ is convex,

(3) $c_{1 B}\|v\|^{2}-c_{2 B} \leq \varphi(z, v, \lambda(z)) \leq c_{3 B}\left(1+\|v\|^{2}\right)$ a.e. on $Z$ for every $[v, \lambda] \in \mathbb{R}^{N} \times B, B \subseteq \Lambda$ compact, with $0<c_{1 B} \leq c_{3 B}<\infty$ and $0 \leq c_{2 B}<\infty$,

(4) for every $x_{1}, x_{2} \in H_{0}^{1}(Z)$ and every $\lambda \in B \subseteq \Lambda, B$ compact, we have

$$
\begin{aligned}
& \int_{Z}\left(a\left(z, D x_{1}(z), \lambda(z)\right)-\left(a\left(z, D x_{2}(z), \lambda(z)\right)\left(D x_{1}(z)-D x_{2}(z)\right) d z\right.\right. \\
& \quad \leq \gamma_{1 B}\left\|x_{1}-x_{2}\right\|_{H_{0}^{1}(z)}^{2}
\end{aligned}
$$

with $\gamma_{1 B}>0, a(z, 0, \lambda)=0$ and for all $x \in H_{0}^{1}(Z) \| a(\cdot, D x(\cdot)$, $\lambda(\cdot) \|_{L^{2}\left(Z, \mathbb{R}^{N}\right)} \leq \gamma_{2 B}\left(1+\|x\|_{H_{0}^{1}(Z)}\right)$ with $\gamma_{2 B}>0$,

(5) if $\lambda_{n} \rightarrow \lambda$ in the metric space $\Lambda$, then $\varphi\left(z, v, \lambda_{n}(z)\right) \rightarrow \varphi(z, v, \lambda(z))$ a.e. on $Z$.

$H(f): \quad f: T \times Z \times \mathbb{R} \times \mathbb{R}^{k} \rightarrow \mathbb{B}$ is a function such that

(1) $(t, z, \lambda) \rightarrow f(t, z, x, \lambda)$ is measurable,

(2) $|f(t, z, x, \lambda(z))-f(t, z, y, \lambda(z))| \leq k_{B}(t, z)|x-y|$ a.e. for all $\lambda \in B \subseteq$ $\Lambda$ compact and $k_{B} \in L^{1}\left(T, L^{\infty}(Z)\right)$,

(3) $|f(t, z, x, \lambda(z))| \leq a_{B}(t, z)+b_{B}|x|$ a.e. for all $\lambda \in B \subseteq \Lambda$ compact and with $a_{B} \in L^{2}(T \times Z), b_{B} \geq 0$,

(4) if $\lambda_{n} \rightarrow \lambda$ in $\Lambda$, then for almost all $t \in T f\left(t, \cdot, x, \lambda_{n}(\cdot)\right) \stackrel{s}{\rightarrow} f(t, \cdot, x, \lambda(\cdot))$ in $L^{2}(Z)$.

REMARK 1. We could have assumed that the controls are $\mathbb{R}^{m}$-valued $m>1$, in which case $f(t, z, x, \lambda)$ is $\mathbb{R}^{m}$-valued and the right-hand side of the partial differential equation becomes $(f(t, z, x(z), \lambda(z)), u(z))_{\mathbb{R}^{m}}$ denoting the Euclidean inner product in $\mathbb{R}^{m}$. However to simplify our notation, we have assumed that $m=1$.

$H(\theta): \quad \theta: T \times Z \times \mathbb{R}^{k} \rightarrow \mathbb{R}_{+}$is a function such that

(1) $\theta(\cdot, \cdot, \lambda(\cdot)) \in L^{\infty}(T \times Z)$ and $\sup \left\{\|\theta(\cdot, \cdot, \lambda(\cdot))\|_{\infty}: \lambda \in B\right\}<\infty$ for any $B \subseteq \Lambda$ compact,

(2) if $\lambda_{n} \rightarrow \lambda$ in $\Lambda$, then for almost all $t \in T, \theta\left(t, \cdot, \lambda_{n}(\cdot)\right) \stackrel{s}{\rightarrow} \theta(t, \cdot, \lambda(\cdot))$ in $L^{2}(Z)$.

$H(L): \quad L: T \times Z \times \mathbb{R} \times \mathbb{R} \times \mathbb{R}^{k} \rightarrow \mathbb{R}$ is an integrand such that

(1) $(t, z, \lambda) \rightarrow L(t, z, x, u, \lambda)$ is measurable,

(2) $|u|^{2} \leq L(t, z, x, u, \lambda(z)) \leq \gamma_{3 B}\left(1+|x|^{2}+|u|^{2}\right)$ a.e. on $T \times Z$, for all $[x, u, \lambda] \in \mathbb{R} \times \mathbb{R} \times B, B \subseteq \Lambda$ compact and with $\gamma_{3 B}>0$, 
(3) for every $x, y \in \mathbb{R}$ such that $|x-y|<1$ and every $\lambda \in B \subseteq \Lambda$ compact $|L(t, z, x, u, \lambda(z))-L(t, z, y, u, \lambda(z))| \leq \rho_{B}(|x-y|)\left(\ell+|x|^{2}+|u|^{2}\right)$ with $\rho_{B}:[0,1) \rightarrow \mathbb{R}$ increasing, continuous and $\rho_{B}(0)=0, \ell \geq 0$,

(4) $u \rightarrow L(t, x, u, \lambda)$ is convex,

(5) if $\lambda_{n} \rightarrow \lambda$ then $L^{*}\left(\cdot, \cdot, x, u^{*}, \lambda_{n}(\cdot)\right) \stackrel{w}{\rightarrow} L^{*}\left(\cdot, \cdot, x, u^{*}, \lambda(\cdot)\right)$ in $L^{1}(T \times Z)$ for every $\left[x, u^{*}\right] \in \mathbb{R} \times \mathbb{R}$; here $L^{*}$ denotes the conjugate function of $L$ with respect to $u$ and $\stackrel{w}{\rightarrow}$ denotes the weak convergence in $L^{1}(T \times Z)$ (recall that $L^{*}\left(t, z, x, u^{*}, \lambda\right)=\sup \left[\left(u^{*}, u\right)_{\mathbb{R}^{k}}-L(t, z, x, u, \lambda): u \in \mathbb{R}^{k}\right]$, with $(\cdot, \cdot)_{\mathbb{R}^{k}}$ being the Euclidean inner product in $\left.\mathbb{R}^{k}\right)$.

REMARK 2. This hypothesis which incorporates the quadratic cost functionals considered by Lions [7], will guarantee the $\Gamma_{\text {seq }}$-convergence of $J\left(x, u, \lambda_{n}\right)=\int_{0}^{b} \int_{Z} L(t$, $\left.z, x(t, z), u(t, z), \lambda_{n}(z)\right) d z d t$ as $\lambda_{n} \rightarrow \lambda$ in $\Lambda$. In particular, assume that the controls take values in $\mathbb{R}^{m}$ and consider the quadratic cost functional

$$
\begin{aligned}
J(x, u, \lambda)= & \int_{0}^{b} \int_{Z}\left|x(t, z)-y_{0}(t, z, \lambda(z))\right|^{2} d z d t \\
& +\frac{1}{2} \int_{0}^{b} \int_{Z}(N(t, z, \lambda(z)) u(t, z), u(t, z))_{\mathbb{R}^{m}} d z d t
\end{aligned}
$$

with $y_{0}(\cdot, \cdot, \lambda(\cdot)) \in L^{2}(T \times Z)$ and $N(\cdot, \cdot, \lambda(\cdot)) \in L^{1}\left(T \times Z, \mathbb{R}^{m \times m}\right)$ for every $\lambda(\cdot) \in \Lambda$. We assume that for every $(t, z) \in T \times Z, N(t, z, \lambda(z))$ is symmetric and positive definite and so $N(t, z, \lambda(z))^{-1}$ exists. Furthermore, we assume that if $\lambda_{n} \rightarrow \lambda$, then $y_{0}\left(\cdot, \cdot, \lambda_{n}(\cdot)\right) \stackrel{w}{\rightarrow} y_{0}(\cdot, \cdot, \lambda(\cdot))^{2}, y_{0}\left(\cdot, \cdot, \lambda_{n}(\cdot)\right)^{2} \stackrel{w}{\rightarrow} y_{0}(\cdot, \cdot, \lambda(\cdot))$ in $L^{1}(T \times Z)$ and $N\left(\cdot, \cdot, \lambda_{n}(\cdot)\right)^{-1} \stackrel{w}{\rightarrow} N(\cdot, \cdot, \lambda(\cdot))^{-1}$ in $L^{1}\left(T \times Z, \mathbb{R}^{m \times m}\right)$. So if we set $L(t, z, x, u, \lambda)=\left|x-y_{0}(t, z, \lambda(z))\right|^{2}+\frac{1}{2}(N(t, z, \lambda(z)) u, u)_{\mathbb{R}^{m}}$, we have that $L^{*}\left(t, z, x, u^{*}, \lambda\right)=\frac{1}{2}\left(N(t, z, \lambda(z))^{-1} u^{*}, u^{*}\right)_{\mathbb{R}^{m}}-\left|x-y_{0}(t, z, \lambda(z))\right|^{2}$ and for this $L$ hypothesis $H(L)$ is satisfied. This type of cost functional was used by Lions [7].

\section{Convergence of the costs and the constraints}

We start with the $\Gamma_{\text {seq-convergence of the costs, which can be obtained directly }}$ from the results of Buttazzo-Dal Maso [2]. Let $H=L^{2}(Z), X=H_{0}^{1}(Z)$ and $X^{*}=H^{-1}(Z)$. From the Sobolev embedding theorem, we know that $\left(X, H, X^{*}\right)$ is an evolution triple. For this triple we will use the notation introduced in Section 2. Denote $J(x, u, \lambda)=\int_{0}^{b} \int_{Z} L(t, z, x(t, z), u(t, z), \lambda(z)) d z d t$, for all $(x, u, \lambda) \in$ $W(T) \times L^{2}(T, H) \times \Lambda$, where $W(T)$ is the Hilbert space introduced in Section 2 .

PROPOSITION 1. If hypothesis $H(L)$ holds and $\lambda_{n} \rightarrow \lambda$ in $\Lambda$, then $\Gamma_{\text {seq }}(\mathbb{N}, w-W(T)$, $\left.w-L^{2}(T, H)^{-}\right) J\left(x, u, \lambda_{n}\right)=J(x, u, \lambda)$. 
Here $w-W(T)$ (respectively, $w-L^{2}(T, H)$ ) denotes the Hilbert space $W(T)$ (respectively, $L^{2}(T, H)=L^{2}(T \times Z)$ ) furnished with the weak topology. The proof of Proposition 1 goes exactly as the proof of Lemma 3.1 of Buttazza-Dal Maso [2], with the independent variable $z \in Z$ replaced by $(t, z) \in T \times Z$, since our system is parabolic and not elliptic. Also recall that $W(T)$ embeds compactly into $L^{2}(T, H)$ (see Section 2), which allows us to consider $W(T)$ with the weak topology instead of $L^{2}(T, H)$, with the strong topology.

Next let $p: H_{0}^{1}(Z) \times H_{0}^{1}(Z) \times \Lambda \rightarrow \mathbb{R}$ be the Dirichlet form defined by

$$
p(x, y, \lambda)=\int_{z} a(z, D x(z), \lambda(z)) D y(z) d z .
$$

From the Cauchy-Schwartz inequality, we have for all $\lambda \in B \subseteq \Lambda$ compact:

$$
\begin{aligned}
|p(x, y, \lambda)| & \leq\|a(\cdot, D x(\cdot), \lambda(\cdot))\|_{L^{2}\left(Z, \mathbb{R}^{N}\right)}\|D y\|_{L^{2}\left(Z, \mathbb{R}^{N}\right)} \\
& \leq \gamma_{2 B}\left(1+\|x\|_{H_{0}^{1}(Z)}\right)\|y\|_{H_{0}^{1}(Z)} \quad(\text { cf. hypothesis } H(a)(4)) .
\end{aligned}
$$

So there exists a generally nonlinear operator $A: X \times \Lambda \rightarrow X^{*}$ defined by

$$
\langle A(x, \lambda), y\rangle=p(x, y, \lambda)
$$

for every $x, y \in X$ and every $\lambda \in \Lambda$. As we indicated in Section $2,\langle\cdot, \cdot\rangle$ denotes the duality brackets for the pair $\left(H_{0}^{1}(Z), H^{-1}(Z)\right)$.

Also let $\hat{f}: T \times H \times \Lambda \rightarrow H$ be the Nemitsky (superposition) operator corresponding to the function $f(t, z, x, \lambda)$; that is, $\hat{f}(t, x, \lambda)(z)=f(t, z, x(z), \lambda(z))$.

Furthermore, let $U(t, \lambda)=\left\{u \in L^{2}(Z):|u(z)| \leq \theta(t, z, \lambda(z))\right.$ a.e. $\}$. Clearly because of hypothesis $H(\theta)$, for every $(t, \lambda) \in T \times \Lambda, U(t, \lambda) \subseteq L^{\infty}(Z)$ and so given any $u \in U(t, \lambda),\left(\hat{f}(t, x, \lambda) u(\cdot) \in L^{2}(Z)\right.$. Note that for every $\lambda \in \Lambda$, the graph of the set-valued map $t \rightarrow U(t, \lambda)$ is given by

$$
G r U(\cdot, \lambda)=\left\{(t, u) \in T \times L^{2}(Z): \int_{C}|u(z)| d z \leq \int_{C} \theta(t, z, \lambda(z)) d z, C \in B(Z)\right\}
$$

with $B(Z)$ being the Borel $\sigma$-field of $Z$.

Let $\xi_{1}(u, C)=\int_{C}|u(z)| d z$ and $\xi_{2}(t, C)=\int_{C} \theta(t, z, \lambda(z)) d z$. Then $u \rightarrow \xi_{1}(u, C)$ is continuous, while by Fubini's theorem $t \rightarrow \xi_{2}(t, C)$ is measurable. Recall that $B(Z)$ is countably generated and so we can find a countable field $\left\{C_{n}\right\}_{n \geq 1} \subseteq B(Z)$ which generates $B(Z)$; that is, $\sigma\left(\left\{C_{n}\right\}_{n \geq 1}\right)=B(Z)$. Define

$$
\xi_{1 n}(u)=\xi_{1}\left(u, C_{n}\right) \text { and } \xi_{2 n}(t)=\xi_{2}\left(t, C_{n}\right) .
$$

We have that

$$
\operatorname{Gr} U(\cdot, \lambda)=\bigcap_{n \geq 1}\left\{(t, u) \in T \times L^{2}(Z): \xi_{1 n}(u) \leq \xi_{2 n}(t)\right\} \in B(T) \times B\left(L^{2}(Z)\right)
$$


with $B(T)$ (respectively, $B\left(L^{2}(Z)\right)$ ) being the Borel $\sigma$-field of $T$ (respectively, of $L^{2}(Z)$ ). So $t \rightarrow U(t, \lambda)$ is a measurable set-valued map (see Wagner [12], Theorem 4.2) and by Aumann's selection theorem (see Wagner [12], Theorem 5.10), it admits measurable selectors (that is, maps $u: T \rightarrow L^{2}(Z)$ measurable such that $u(t) \in U(t, \lambda)$ for all $t \in T$ ).

Then we can rewrite the dynamics of our optimal control problem (1), in the following equivalent evolution equation form:

$$
\left\{\begin{array}{c}
\dot{x}(t)+A(x(t), \lambda)+\hat{f}(t, x(t), \lambda) u(t), \\
x(0)=\hat{x}_{0}, \\
u(t) \in U(t, \lambda) \text { a.e., } u(\cdot) \text { measurable, }
\end{array}\right\}
$$

where $\hat{x}_{0}=x_{0}(\cdot) \in L^{2}(Z)$.

By admissible "state-control" pair of (2) (equivalently of (1)), we mean a pair $[x, u] \in W(T) \times L^{2}(T, H)$ satisfying (2) (equivalently the constraints of (1)). Given $\lambda \in \Lambda$, let $\Delta(\lambda) \subseteq W(T) \times L^{2}(T, H)$ be the set of admissible state-control pairs corresponding to this particular choice of the parameter. Let $\delta_{\Delta(\lambda)}(x, u)$ be the indicator function of $\Delta(\lambda)$; that is, $\delta_{\Delta(\lambda)}(x, u)=0$ if $[x, u] \in \Delta(\lambda)$ and $+\infty$ otherwise. Then problem (1) can be rewritten in the equivalent unconstrained form

$$
m(\lambda)=\inf \left[J(x, u, \lambda)+\delta_{\Delta(\lambda)}(x, u)\right] .
$$

In the next proposition, we establish the $\Gamma_{\text {seq }}$-convergence of the sets of admissible state-control pairs.

Directly from the definition of $\Gamma_{\text {seq }}$-convergence, we have that if $V_{1}, V_{2}$ are topological spaces and $B_{n} \subseteq V_{1} \times V_{2}, n \geq 1$, then

$$
\Gamma_{\mathrm{seq}}\left(\mathbb{N}, V_{1}, V_{2}^{-}\right) \delta_{B_{n}}(x, y)=\delta_{B}(x, y)
$$

if and only if the following two conditions hold:

(i) if $x_{n} \rightarrow x$ in $V_{1}, y_{n} \rightarrow y$ in $V_{2}$ and $\left(x_{n}, y_{n}\right) \in B_{n}$ for infinitely many $n$ 's, then $(x, y) \in B$

(ii) if $(x, y) \in B$ and $x_{n} \rightarrow x$ in $V_{1}$, then there exist $\left\{y_{n}\right\}_{n \geq 1} \subseteq V_{2}$ and $n_{0} \geq 1$ such that $y_{n} \rightarrow y$ in $V_{2}$ and $\left(x_{n}, y_{n}\right) \in B_{n}$ for all $n \geq n_{0}$

(see also Buttazza-Dal Maso [2], p. 388).

REMARK 3. If $V_{1}, V_{2}$ are first countable and condition (ii) above is replaced by the weaker condition

(ii) ${ }^{\prime}$ if $(x, y) \in B$, then there exists a sequence $\left\{\left(x_{n}, y_{n}\right)\right\}_{n \geq 1} \subseteq V_{1} \times V_{2}$ such that for all $n \geq 1\left(x_{n}, y_{n}\right) \in B_{n}$ and $x_{n} \rightarrow x, y_{n} \rightarrow y$ as $n \rightarrow \infty$, 
then we have a characterization of the classical Kuratowski convergence of sets (see Dal Maso [3], p. 41).

We will use the above observation concerning the $\Gamma_{\text {seq }}$-convergence of indicator functions, to establish the $\Gamma_{\text {seq }}$-limit of $\left\{\delta_{\Delta\left(\lambda_{n}\right)}(\cdot, \cdot)\right\}_{n \geq 1}$.

PROPOSITION 2. If hypotheses $H(a), H(f), H(\theta)$ fold and $\lambda_{n} \rightarrow \lambda$ in $\Lambda$, then $\Gamma_{\text {seq }}\left(\mathbb{N}, w-W(T)^{-}, w-L^{2}(T, H)\right) \delta_{\Delta\left(\lambda_{n}\right)}(x, u)=\delta_{\Delta(\lambda)}(x, u)$.

ProOF. Let $[x, u] \in \Delta(\lambda)$ and $u_{n} \stackrel{w}{\rightarrow} u$ in $L^{2}(T, H), u_{n}(t) \in U\left(t, \lambda_{n}\right)$ a.e. Let $x_{n}(\cdot) \in W(T)$ be the unique trajectory generated by the control $u_{n}(\cdot)$ (see Papageorgiou [8], Theorem 3.4). Uniqueness follows from the (strong) monotonicity of $A\left(\cdot, \lambda_{n}\right)$ (cf. hypothesis $H(a)(4))$ and the Lipschitzness of $\hat{f}\left(t, \cdot, \lambda_{n}\right) u_{n}$ (cf. hypothesis $H(f)(2)$ ). So we have

$$
\begin{gathered}
\left.\dot{x}_{n}(t)+A\left(x_{n}(t), \lambda_{n}\right)=\hat{f}\left(t, x_{n}(t), \lambda_{n}\right) u_{n}\right) \text { a.e. } \\
x_{n}(0)=\hat{x}_{0} \\
u_{n}(t) \in U\left(t, \lambda_{n}\right) \text { a.e. }
\end{gathered}
$$

We will derive some a priori bounds for the $x_{n}$ 's. First let $B=\left\{\lambda_{n}, \lambda\right\}_{n \geq 1} \subseteq \Lambda$ compact. Then from hypothesis $H(a)(4)$, we have for $\lambda^{\prime} \in B$ :

$$
\begin{gathered}
\left\langle\left(A\left(x, \lambda^{\prime}\right)-A\left(y, \lambda^{\prime}\right), x-y\right\rangle \geq \hat{\gamma}_{B}\|x-y\|^{2}, \hat{\gamma}_{B}>0,\right. \\
\left\langle\left(A\left(x, \lambda^{\prime}\right), x\right\rangle \geq \hat{\gamma}_{B}^{\prime}\|x\|^{2}, \hat{\gamma}_{B}^{\prime}>0 \text { and }\|A(x, \lambda)\|_{*} \leq \hat{\gamma}_{B}^{\prime \prime}(1+\|x\|), \hat{\gamma}_{B}^{\prime \prime}>0,\right.
\end{gathered}
$$

where $\|\cdot\|$ (respectively, $\|\cdot\|_{*}$ ) denotes the norm of $H_{0}^{1}(Z)$ (respectively, $H^{-1}(Z)$ ). Also $\langle\cdot, \cdot\rangle$ denotes the duality brackets for the pair $\left(X=H_{0}^{1}(Z), X^{*}=H^{-1}(Z)\right)$, while in what follows by $(\cdot, \cdot)$ we will denote the inner product in $H=L^{2}(Z)$ and by $|\cdot|$ the corresponding norm. Recall that $\left.\langle\cdot, \cdot\rangle\right|_{X \times H}=(\cdot, \cdot)$; see Section 2 . Then we have

$$
\begin{aligned}
& \left\langle\dot{x}_{n}(t), x_{n}(t)\right\rangle+\left\langle A\left(x_{n}(t), \lambda_{n}\right), x_{n}(t)\right\rangle=\left(\hat{f}\left(t, x_{n}(t), \lambda_{n}\right) u_{n}(t), x_{n}(t)\right) \text { a.e. } \\
& \Rightarrow \quad \frac{1}{2} \frac{d}{d t}\left|x_{n}(t)\right|^{2}+\hat{\gamma}_{B}^{\prime}\left\|x_{n}(t)\right\|^{2} \leq\left\|\hat{f}\left(t, x_{n}(t), \lambda_{n}\right) u_{n}(t)\right\|_{*}\left\|x_{n}(t)\right\|^{2} \text { a.e. }
\end{aligned}
$$

Applying on the right-hand side Cauchy's inequality with $\epsilon>0$, we get

$$
\frac{1}{2} \frac{d}{d t}\left|x_{n}(t)\right|^{2}+\hat{\gamma}_{B}^{\prime}\left\|x_{n}(t)\right\|^{2} \leq \frac{\epsilon}{2}\left\|\hat{f}\left(t, x_{n}(t), \lambda_{n}\right) u_{n}(t)\right\|_{*}^{2}+\frac{1}{2 \epsilon}\left\|x_{n}(t)\right\|^{2} \text { a.e. }
$$


Let $\epsilon=1 / 2 \hat{\gamma}_{B}^{\prime}$. We have

$$
\begin{gathered}
\quad \frac{1}{2} \frac{d}{d t}\left|x_{n}(t)\right|^{2} \leq \frac{1}{4 \hat{\gamma}_{B}^{\prime}}\left\|\hat{f}\left(t, x_{n}(t), \lambda_{n}\right) u_{n}(t)\right\|_{*}^{2} \\
\Rightarrow\left|x_{n}(t)\right|^{2} \leq\left|\hat{x}_{0}\right|^{2}+\frac{1}{2 \hat{\gamma}_{B}^{\prime}} \int_{0}^{t}\left\|\hat{f}\left(s, x_{n}(s), \lambda_{n}\right) u_{n}(s)\right\|_{*}^{2} d s \\
\leq\left|\hat{x}_{0}\right|^{2}+\frac{1}{2 \hat{\gamma}_{B}^{\prime}} \int_{0}^{t}\left|\hat{f}\left(s, x_{n}(s), \lambda_{n}\right) u_{n}(s)\right|^{2} d s \\
\leq\left|\hat{x}_{0}\right|^{2}+\frac{1}{2 \hat{\gamma}_{B}^{\prime}}\left(\int_{0}^{t}\left|\hat{f}\left(s, x_{n}(s), \lambda_{n}\right) u_{n}(s)\right|^{2} d s\right) \sup _{n \geq 1}\left\|\theta\left(\cdot, \cdot, \lambda_{n}\right)\right\|_{\infty}^{2} .
\end{gathered}
$$

From hypothesis $H(\theta)(1)$, we have that $\sup _{n \geq 1}\left\|\theta\left(\cdot, \cdot, \lambda_{n}\right)\right\|_{\infty}^{2}<\infty$. So

$$
\left|x_{n}(t)\right|^{2} \leq\left|\hat{x}_{0}\right|^{2}+\mu_{B} \int_{0}^{t}\left(2 \hat{a}_{B}(s)^{2}+2 b_{B}^{2}\left|x_{n}(s)\right|^{2}\right) d s
$$

with $\mu_{B}>0, \hat{a}_{B}(s)=\left\|a_{B}(s, \cdot)\right\|_{2}$ and $b_{B}>0$ as in hypothesis $H(f)(3)$.

Invoking Gronwall's lemma, we deduce that there exists $M_{1}>0$ such that for all $n \geq 1$ and all $t \in T$ we have

$$
\left|x_{n}(t)\right| \leq M_{1} .
$$

Next in inequality (3) above, let $\epsilon=1 / \hat{\gamma}_{B}^{\prime}$. We get

$$
\begin{gathered}
\frac{1}{2} \frac{d}{d t}\left|x_{n}(t)\right|^{2}+\frac{\hat{\gamma}_{B}^{\prime}}{2}\left\|x_{n}(t)\right\|^{2} \leq \frac{1}{2 \hat{\gamma}_{B}^{\prime}}\left|\hat{f}\left(t, x_{n}(t), \lambda_{n}\right) u_{n}(t)\right|^{2} \text { a.e. } \\
\Rightarrow \hat{\gamma}_{B}^{\prime} \int_{0}^{t}\left\|x_{n}(x)\right\|^{2} d s \leq\left|\hat{x}_{0}\right|^{2}+\frac{1}{\hat{\gamma}_{B}^{\prime}} \sup _{n \geq 1}\left\|\theta\left(\cdot, \cdot, \lambda_{n}\right)\right\|_{\infty}^{2} \int_{0}^{t}\left(2 \hat{a}_{B}(s)^{2}+2 b_{B}^{2}\left|x_{n}(s)\right|^{2}\right) d s .
\end{gathered}
$$

Using bound (4) above, we deduce that there exists $M_{2}>0$ such that for all $n \geq 1$ we have

$$
\left\|x_{n}(t)\right\|_{L^{2}(T, X)} \leq M_{2} .
$$

Finally using hypothesis $H(a)(4)$ and $H(f)(3)$, as well as bounds (4) and (5), we conclude that there exists $M_{3}>0$ such that for all $n \geq 1$ we have

$$
\left\|\dot{x}_{n}(t)\right\|_{L^{2}\left(T, X^{*}\right)} \leq M_{3}
$$

From (5) and (6) above, we deduce that $\left\{x_{n}\right\}_{n \geq 1}$ is bounded in $W(T)$, hence relatively weakly sequentially compact. So by passing to a subsequence if necessary, we may assume that $x_{n} \stackrel{w}{\rightarrow} \hat{x}$ in $W(T)$. 
Next let $g(t)=\hat{f}(t, x(t), \lambda) u(t)$ and let $y_{n}(\cdot) \in W(T)$ be the unique solution of the following evolution equation

$$
\begin{gathered}
\dot{y}_{n}(t)+A\left(t, y_{n}(t), \lambda_{n}\right)=g(t) \text { a.e., } \\
y_{n}(0)=\hat{x}_{0} .
\end{gathered}
$$

The existence and uniqueness of $y_{n}(\cdot) \in W(T)$ solving the above Cauchy problem, is guaranteed by Theorem 30.A, p. 771 of Zeidler [13]. Let $\mathscr{U}$ be the family of all open subsets of $Z$ and define the functional $\Phi: H_{0}^{1}(Z) \times \mathscr{U} \times \Lambda \rightarrow \mathbb{R}$ by $\Phi\left(x, Z^{\prime}, \lambda\right)=\int_{Z^{\prime}} \varphi(z, D x(z), \lambda(z)) d z$. Then from hypothesis $H(a)(5)$, together with Theorem 5.14, p. 51 and Proposition 8.10, p. 93 of Dal Maso [3], we have that $\Gamma_{\mathrm{seq}}\left(\mathbb{N}, w-H_{0}^{1}\left(Z^{\prime}\right)\right) \Phi\left(x, Z^{\prime}, \lambda_{n}\right)=\Phi\left(x, Z^{\prime}, \lambda\right)$. Hence invoking Theorem 3.2 of Defranceschi [5], we get that $A\left(\cdot, \lambda_{n}\right) \stackrel{G}{\rightarrow} A(\cdot, \lambda)$. Then Theorem 1 of Kolpakov [6], tells us that $y_{n} \stackrel{w}{\rightarrow} x$ in $W(T)$. Exploiting the monotonicity of the operator $A\left(\cdot, \lambda_{n}\right)$, we have

$$
\begin{aligned}
\left\langle\dot{x}_{n}(t)-\right. & \left.\dot{y}_{n}(t), x_{n}(t)-y_{n}(t)\right\rangle \leq\left(\hat{f}\left(t, x_{n}(t), \lambda_{n}\right) u_{n}(t)-g(t), x_{n}(t)-y_{n}(t)\right) \text { a.e. } \\
= & \left(\hat{f}\left(t, x_{n}(t), \lambda_{n}\right) u_{n}(t)-\left(\hat{f}\left(t, x(t), \lambda_{n}\right) u_{n}(t), x_{n}(t)-y_{n}(t)\right)\right. \\
& +\left(\hat{f}\left(t, x(t), \lambda_{n}\right) u_{n}(t)-\hat{f}(t, x(t), \lambda) u(t), x_{n}(t)-y_{n}(t)\right) \text { a.e. } \\
\Rightarrow & \frac{1}{2}\left|x_{n}(t)-y_{n}(t)\right|^{2} \\
\leq & \int_{0}^{t}\left(\hat{f}\left(s, x_{n}(s), \lambda_{n}\right) u_{n}(s)-\hat{f}\left(s, x(s), \lambda_{n}\right) u_{n}(s), x_{n}(s)-y_{n}(s)\right) d s \\
& \quad+\int_{0}^{t}\left(\hat{f}\left(s, x(s), \lambda_{n}\right) u_{n}(s)-\hat{f}(s, x(s), \lambda) u(s), x_{n}(s)-y_{n}(s)\right) d s \\
\leq & \int_{0}^{t}\left|\hat{f}\left(s, x_{n}(s), \lambda_{n}\right) u_{n}(s)-\hat{f}\left(s, x(s), \lambda_{n}\right) u_{n}(s)\right| \cdot\left|x_{n}(s)-y_{n}(s)\right| d s \\
& +\int_{0}^{t}\left(\hat{f}\left(s, x(s), \lambda_{n}\right) u_{n}(s)-\hat{f}(s, x(s), \lambda) u(s), x_{n}(s)-y_{n}(s)\right) d s
\end{aligned}
$$

Observe that

$$
\begin{aligned}
& \int_{0}^{t}\left|\hat{f}\left(s, x_{n}(s), \lambda_{n}\right) u_{n}(s)-\hat{f}\left(s, x(s), \lambda_{n}\right) u_{n}(s)\right| \cdot\left|x_{n}(s)-y_{n}(s)\right| d s \\
& \quad \leq \sup _{n \geq 1}\left\|\theta\left(\cdot, \cdot, \lambda_{n}\right)\right\|_{\infty} \int_{0}^{t}\left|\hat{f}\left(s, x_{n}(s), \lambda_{n}\right) u_{n}(s)-\hat{f}\left(s, x(s), \lambda_{n}\right) u_{n}(s)\right| \cdot\left|x_{n}(s)-y_{n}(s)\right| d s \\
& \quad \leq \sup _{n \geq 1}\left\|\theta\left(\cdot, \cdot, \lambda_{n}\right)\right\|_{\infty} \int_{0}^{t} \hat{k}_{B}(s)\left|x_{n}(s)-y_{n}(s)\right|^{2} d s, \text { with } \hat{k}_{B}(s)=\left\|k_{B}(s, \cdot)\right\|_{\infty} .
\end{aligned}
$$


Also we have

$$
\begin{aligned}
& \int_{0}^{t}\left(\hat{f}\left(s, x(s), \lambda_{n}\right) u_{n}(s)-\hat{f}(s, x(s), \lambda) u(s), x_{n}(s)-y_{n}(s)\right) d s \\
& \quad+\int_{0}^{t} \int_{Z}\left(f\left(s, z, x(s, z), \lambda_{n}(z)\right) u_{n}(s, z)\right. \\
& \quad-f(s, z, x(s, z), \lambda(z))) u(s, z))\left(x_{n}(s, z)-y_{n}(s, z)\right) d z d s \\
& =\int_{0}^{t} \int_{Z} f\left(s, z, x(s, z), \lambda_{n}(z)\right)\left(u_{n}(s, z)-u(s, z)\right)\left(x_{n}(s, z)-x(s, z)\right) d z d s \\
& \quad+\int_{0}^{t} \int_{Z}\left(f\left(s, z, x(s, z), \lambda_{n}(z)\right)\right. \\
& \quad-f(s, z, x(s, z), \lambda(z))) u(s, z)\left(x_{n}(s, z)-y_{n}(s, z)\right) d z d s .
\end{aligned}
$$

Since $W(T)$ embeds into $L^{2}(T, H)$ compactly, we have that $x_{n} \stackrel{s}{\rightarrow} \hat{x}$ and $y_{n} \stackrel{s}{\rightarrow} x$ in $L^{2}(T, H)=L^{2}(T \times Z)$. Also by hypothesis $u_{n} \stackrel{w}{\rightarrow} u$ in $L^{2}(T \times Z)$ and because of hypothesis $H(\theta)$, we have $u_{n} \stackrel{\omega^{*}}{\rightarrow} u$ in $L^{\infty}(T \times Z)$. So using hypothesis $H(f)$, we get

$$
\begin{aligned}
& \int_{0}^{t} \int_{Z} f\left(s, z, x(s, z), \lambda_{n}(z)\right)\left(u_{n}(s, z)-u(s, z)\right)\left(x_{n}(s, z)-x(s, z)\right) d z d s \\
& \left.\quad+\int_{0}^{t} \int_{Z}\left(f\left(s, z, x(s, z), \lambda_{n}(z)\right)-f(s, z, x(s, z), \lambda(z))\right) u(s, z)\right)\left(x_{n}(s, z)\right. \\
& \left.\quad-y_{n}(s, z)\right) d z d s \rightarrow 0 \text { as } n \rightarrow \infty \\
& \Rightarrow \int_{0}^{t}\left(\hat{f}\left(s, x(s), \lambda_{n}\right) u_{n}(s)-\hat{f}(s, x(s), \lambda) u(s), x_{n}(s)-y_{n}(s)\right) d s \rightarrow 0 \quad \text { as } n \rightarrow \infty .
\end{aligned}
$$

Thus in the limit as $n \rightarrow \infty$, we get

$$
|\hat{x}(t)-x(t)|^{2} \leq 2 \sup _{n \geq 1}\left\|\theta\left(\cdot, \cdot, \lambda_{n}\right)\right\|_{\infty} \int_{0}^{t} \hat{k}_{B}(s)\left|\hat{x}_{n}(s)-x(s)\right|^{2} d s .
$$

From Gronwall's lemma, we conclude that $x=\hat{x}$. Hence every subsequence of $\left\{x_{n}\right\}_{n \geq 1}$ has a further subsequence which weakly converges in $W(T)$ to $x$. Since ${\overline{\left\{x_{n}(\cdot)\right\}_{n \geq 1}}}^{w}$ equipped with the relative weak-W(T) topology is metrizable, we conclude that $x_{n} \stackrel{w}{\rightarrow} x$ in $W(T)$ and $\left[x_{n}, u_{n}\right] \in \Delta\left(\lambda_{n}\right), n \geq 1$. So we have established condition (ii) in the characterization of $\Gamma_{\text {seq }}\left(\mathbb{N}, w-W(T)^{-}, w-L^{2}(T, H)\right) \delta_{\Delta\left(\lambda_{n}\right)}(\cdot, \cdot)$ provided earlier.

Next we will show that condition (i) is also valid, establishing this way the desired $\Gamma_{\text {seq }}$-convergence of the indicator maps $\delta_{\Delta\left(\lambda_{n}\right)}(\cdot, \cdot)$. So let $\left[x_{n}, u_{n}\right] \in \Delta\left(\lambda_{n}\right), n \geq 1$, and assume that $x_{n} \stackrel{w}{\rightarrow} x$ in $W(T)$ and $u_{n} \stackrel{w}{\rightarrow} u$ in $L^{2}(T, H)$. We will show that $[x, u] \in \Delta(\lambda)$. 
Let $g_{n}(t)=\hat{f}\left(t, x_{n}(t), \lambda_{n}\right) u_{n}(t)$ and $g(t)=\hat{f}(t, x(t), \lambda) u(t)$. We have already seen in the first part of the proof, that by passing to a subsequence if necessary, we may have $g_{n} \stackrel{w}{\rightarrow} g$ in $L^{2}(T, H)$. Let $v_{n} \in W(T)$ be the unique solution of the evolution equation

$$
\begin{gathered}
\dot{v}_{n}(t)+A\left(v_{n}(t), \lambda_{n}\right)=g(t) \quad \text { a.e. } \\
v_{n}(0)=\hat{x}_{0} .
\end{gathered}
$$

Since $A\left(\cdot, \lambda_{n}\right) \stackrel{G}{\rightarrow} A(\cdot, \lambda)$, from Theorem 1 of Kolpakov [6], we know that $v_{n} \stackrel{w}{\rightarrow} v$ in $W(T)$, with $v(\cdot) \in W(T)$ being the unique solution of the evolution equation

$$
\begin{gathered}
\dot{v}(t)+A(v(t), \lambda)=g(t) \quad \text { a.e. } \\
v(0)=\hat{x}_{0} .
\end{gathered}
$$

Recalling that $W(T)$ embeds compactly in $L^{2}(T, H)$, and continuously in $C(T, H)$, by passing to a subsequence if necessary, we may assume that $x_{n}(t) \rightarrow x(t)$ and $v_{n}(t) \rightarrow v(t)$ in $H$ for all $t \in T$ (in fact using the results of Simon [9], we can actually show that $\left\{x_{n}\right\}_{n \geq 1},\left\{v_{n}\right\}_{n \geq 1}$ are relatively compact in $C(T, H)$ ). Exploiting the monotonicity of $A\left(\cdot, \lambda_{n}\right)$ we have

$$
\begin{aligned}
& \quad\left\langle\dot{x}_{n}(t)-\dot{v}_{n}(t), x_{n}(t)-v_{n}(t)\right\rangle \leq\left(g_{n}(t)-g(t), x_{n}(t)-v_{n}(t)\right) \quad \text { a.e. } \\
& \Rightarrow \frac{1}{2} \frac{d}{d t}\left|x_{n}(t)-v_{n}(t)\right|^{2} \leq\left(g_{n}(t)-g(t), x_{n}(t)-v_{n}(t)\right) \quad \text { a.e. } \\
& \Rightarrow \frac{1}{2}\left|x_{n}(t)-v_{n}(t)\right|^{2} \\
& \quad \leq \int_{0}^{t}\left[\left(g_{n}(s)-g(s), x_{n}(s)-x(s)\right)+\left(g_{n}(s)-g(s), x(s)-v(s)\right)\right. \\
& \quad \quad \quad+\left(g_{n}(s)-g(s), v(s)-v_{n}(s)\right) d s \\
& \Rightarrow\left|x_{n}(t)-v_{n}(t)\right| \rightarrow 0 \\
& \Rightarrow|x(t)-v(t)|=0 ; \quad \text { that is, } \quad x=v .
\end{aligned}
$$

Therefore $[x, v] \in \Delta(\lambda)$ and so we have established condition (i) and we can conclude that $\Gamma_{\text {seq }}\left(\mathbb{N}, w-W(T)^{-}, w-L^{2}(T, H)\right) \delta_{\Delta\left(\lambda_{n}\right)}(x, u)=\delta_{\Delta(\lambda)}(x, u)$.

\section{Main convergence theorem}

In this section, using the auxiliary proposition of Section 3, we will examine the variational stability (sensitivity) of our optimal control problem (1).

Let $Q(\lambda)$ be the optimal state-control pairs corresponding to the parameter $\lambda \in \Lambda$; that is, $Q(\lambda)=\left\{[x, u] \in W(T) \times L^{2}(T, H)\right):[x, u]$ solves problem (1) $\}$. Recall that 
if $Y, Z$ are Hausdorff topological spaces, then a set-valued function $R: Y \rightarrow 2^{Z}\{\emptyset\}$ is said to be upper-semicontinuous (u.s.c.), if for all $U$ open in $Z, R^{+}(U)=\{y \in$ $Y: R(y) \subseteq U$ \} is open in $Y$. An u.s.c. $R(\cdot)$ with closed values has a closed graph; that is, if $\left[y_{\beta}, z_{\beta}\right]$ is a net in $Y \times Z, z_{\beta} \in R\left(y_{\beta}\right)$ and $\left[y_{\beta}, z_{\beta}\right] \rightarrow[y, z]$, then we have $z \in R(y)$.

THEOREM 1. If hypotheses $H(A), H(f), H(\theta)$ and $H(L)$ hold, then for every $\lambda \in \Lambda$, $Q(\lambda) \neq \emptyset, \lambda \rightarrow Q(\lambda)$ is u.s.c. from $\Lambda$ into the nonempty, weakly compact subsets of $W(T) \times L^{2}(T, H)$ and $m: \Lambda \rightarrow \mathbb{R}$ is continuous.

PROOF. From the a priori bounds established in the proof of Proposition 2, we know that for every $\lambda \in \Lambda, \Delta(\lambda)$ is weakly sequentially, compact in $W(T) \times L^{2}(T, H)$. Also if $\lambda_{n}=\lambda n \geq 1$ (constant sequence), we have from Proposition 1 that $J(\cdot, \cdot, \lambda)$ is the $\Gamma$-regularization of itself and so from Proposition 1.3.1, p. 16 of Buttazzo [1] we get that $J(\cdot, \cdot, \lambda)$ is sequentially weakly lower semicontinuous on $W(T) \times L^{2}(T, H)$. So for every $\lambda \in \Lambda$, the problem $\inf [J(x, u, \lambda):[x, u] \in \Delta(\lambda)]$ has the solution; that is, $Q(\lambda) \neq \emptyset$.

Next let $\lambda_{n} \rightarrow \lambda$ in $\Lambda$. Combining Propositions 1 and 2 of this paper with Theorem 2.1 of Buttazzo-Dal Maso [2], we get that $w-\overline{\lim } Q\left(\lambda_{n}\right)=\{[x, u] \in W(T) \times$ $\left.L^{2}(T, H)\right):[x, u]=w-\lim \left[x_{n_{k}}, u_{n_{k}}\right],\left[x_{n_{k}}, u_{n_{k}}\right] \in Q\left(\lambda_{n_{k}}\right), n_{1}<n_{2}<n_{3}<\cdots<$

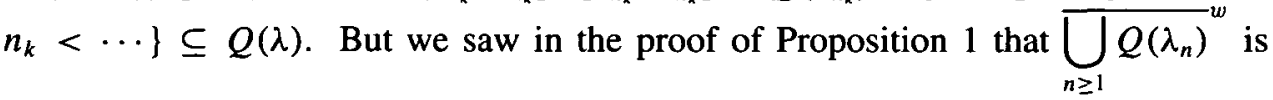
a weakly compact subset of $W(T) \times L^{2}(T, H)$ (recall that the weak topology on the product space $W(T) \times L^{2}(T, H)$ is the product of the weak topologies; that is, $\left.\left(W(T) \times L^{2}(T, H)\right)_{w}=W(T)_{w} \times L^{2}(T, H)_{w}\right)$. So from Remark 1.6. of DeBlasiMyjak [4], we get the desired upper-semicontinuity of $\lambda \rightarrow Q(\lambda)$.

Next let $\lambda_{n} \rightarrow \lambda$ in $\Lambda$ and let $\left[x_{n}, u_{n}\right] \in Q\left(\lambda_{n}\right) n \geq 1$. Then $m\left(\lambda_{n}\right)=J\left(x_{n}, u_{n}, \lambda_{n}\right)$ and by passing to a subsequence if necessary, we may assume that $x_{n} \stackrel{w}{\rightarrow} x$ in $W(T)$ and $u_{n} \stackrel{w}{\rightarrow} u$ in $L^{2}(T, H)$. Then from Proposition 2 we have $[x, u] \in Q(\lambda)$. Also from Proposition 1 and the definition of $\Gamma_{\text {seq }}$-limits, we have

$$
\begin{gathered}
J(x, u, \lambda) \leq \underline{\lim } J\left(x_{n}, u_{n}, \lambda_{n}\right)=\underline{\lim } m\left(\lambda_{n}\right) \\
\Rightarrow m(\lambda) \leq \underline{\lim m\left(\lambda_{n}\right) .}
\end{gathered}
$$

Next let $[x, u] \in Q(\lambda)$ and $\epsilon>0$. We have $m(\lambda)=J(x, u, \lambda),[x, u] \in \Delta(\lambda)$. From Propositions 1 and 2 of this paper and Corollary 2.1 of Buttazzo-Dal Maso [2], we have that

$$
\begin{gathered}
\Gamma_{\mathrm{seq}}\left(\mathbb{N}, w-W(T)^{-}, w-L^{2}(T, H)^{-}\right)\left(J\left(\cdot, \cdot, \lambda_{n}\right)+\delta_{\Delta\left(\lambda_{n}\right)}(\cdot, \cdot)\right)(x, u) \\
=\left(J(\cdot, \cdot, \lambda)+\delta_{\Delta(\lambda)}(\cdot, \cdot)\right)(x, u) .
\end{gathered}
$$


Then from the definition of $\Gamma_{\text {seq }}$-limits we know that we can find a sequence $\left\{\left[x_{n}, u_{n}\right]\right\}_{n \geq 1} \subseteq W(T) \times L^{2}(T, H)$ such that $x_{n} \stackrel{w}{\rightarrow} x$ in $W(T), u_{n} \stackrel{w}{\rightarrow} x$ in $L^{2}(T, H)$ and

$$
\varlimsup
$$

Hence for all $n$ large enough, we have that $\left[x_{n}, u_{n}\right] \in \Delta\left(\lambda_{n}\right)$ and so

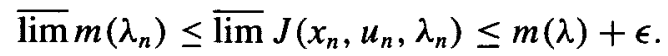

Let $\epsilon \downarrow 0$. We get that

$$
\varlimsup i m\left(\lambda_{n}\right) \leq m(\lambda)
$$

From (7) and (8) above, we deduce that $m\left(\lambda_{n}\right) \rightarrow m(\lambda)$ and so we have proved that $\lambda \rightarrow m(\lambda)$ is continuous.

\section{5: Semilinear systems}

In this section we consider systems with semilinear dynamics. The linearity of the partial differential operator $x \rightarrow A(x, \lambda)$ allows us to incorporate in the framework of this paper, semilinear systems with weakly convergent coefficients (e.g. rapidly oscillating coefficients).

So let $T$ and $Z \subseteq \mathbb{R}^{N}$ be as before. We consider the optimal control problem

$$
\begin{gathered}
\int_{0}^{r} \int_{Z} L(t, z, x(t, z), u(t, z), \lambda(z)) d z d t \rightarrow \inf =m(\lambda), \\
\text { s.t. } \frac{\partial x}{\partial t}-\sum_{i, j=1}^{N} D_{i}\left(a_{i j}(z, \lambda(z)) D_{j} x(t, z)\right) \\
=f(t, z, x(t, z), \lambda(z)) u(t, z) \text { a.e. on } T \times Z, \\
\left.x\right|_{T \times \Gamma}=0, x(0, z)=x_{0}(z) \text { a.e. on } Z, x_{0}(\cdot) \in L^{2}(Z), \\
|u(z)| \leq \theta(t, z, \lambda(z)) \text { a.e. }
\end{gathered}
$$

We will make the following hypothesis on the coefficients, $a_{i j}(z, \lambda)$ :

$\underline{H(a)_{1}}$ for every $\lambda \in \Lambda, a_{i j}(\cdot, \lambda(\cdot)) \in L^{\infty}(Z), a_{i j}(\cdot, \lambda(\cdot))=a_{j i}(\cdot, \lambda(\cdot)) i, j=$ $1,2, \ldots, N$, for every $z \in \mathbb{R}^{N}, m_{1}\|z\|^{2} \leq \sum_{i, j=1}^{N} a_{i j}(z, \lambda(z)) z_{i} z_{j} \leq m_{2}\|z\|^{2}$ with $0<m_{1} \leq m_{2}<\infty$ and if $\lambda_{n} \rightarrow \lambda$ in $\Lambda$, then $a_{i j}\left(\cdot, \lambda_{n}(\cdot)\right) \stackrel{w}{\rightarrow}$ $a_{i j}(\cdot, \lambda(\cdot))$ in $L^{2}(Z)$ for all $i, j \in\{1,2, \ldots, N\}$ and $\sum_{i=1}^{N} D_{i} a_{i j}\left(\cdot, \lambda_{n}(\cdot)\right) \stackrel{s}{\rightarrow}$ $\sum_{i=1}^{N} D_{i} a_{i j}(\cdot, \lambda(\cdot))$ in $H^{-1}(Z)$ for all $j \in\{1,2, \ldots, N\}$. 
In this case $\varphi(z, x, \lambda)=(A(z, \lambda) x, x)_{\mathbb{R}^{N}}$ where $A(z, \lambda)=\left(a_{i j}(z, \lambda)\right)_{i, j=1}^{N} \in \mathbb{R}^{N \times N}$. Let $\Phi: H_{0}^{1}(Z) \times \mathscr{U} \times \Lambda \rightarrow \mathbb{R}$ be $\Phi\left(x, Z^{\prime}, \lambda\right)=\int_{Z} \varphi(z, x(z), \lambda(z)) d z$, where as before (see the proof of Proposition 1), $\mathscr{U}$ is the collection of all open subsets of $Z$. Then because of hypothesis $H(a)_{1}$, we have that if $\lambda_{n} \rightarrow \lambda$ in $\Lambda$, then $\Gamma_{\text {seq }}\left(\mathbb{N}, w-H_{0}^{1}(Z)\right) \Phi\left(x, \lambda_{n}\right)=\Phi(x, \lambda)$ (see Dal Maso [3]). So if for $\lambda^{\prime} \in \Lambda$ we define $\hat{A}\left(\lambda^{\prime}\right) \in \mathscr{L}\left(H_{0}^{\prime}(Z), H^{-1}(Z)\right)$ by $\left\langle\hat{A}\left(\lambda^{\prime}\right) x, y\right\rangle=\int_{z} \sum_{i, j=1}^{N} a_{i j}\left(z, \lambda^{\prime}(z)\right) D_{i} x(z) D_{j} y(z)$ $d z$, then from Theorem 3.2 of Defranceschi [5], we have that $\hat{A}\left(\lambda_{n}\right) \stackrel{G}{\rightarrow} \hat{A}(\lambda)$. Hence the proof of Proposition 2 goes through and therefore we can state the following result.

THEOREM 2. If hypotheses $H(a)_{1}, H(f), H(\theta)$ and $H(L)$ hold, then for all $\lambda \in \Lambda$, $Q(\lambda) \neq \emptyset$, the set-valued map $\lambda \rightarrow Q(\lambda)$ is u.s.c. from $\Lambda$ into the nonempty, weakly contact subsets of $W(T) \times L^{2}(T, H)$ and $m: \Lambda \rightarrow \mathbb{R}$ is continuous.

As a simple illustration, let $Z \subseteq \mathbb{R}^{2}$ and assume that the sequence of partial differential operators of the approximating problems is $B_{n}=-\Delta-\frac{1}{2} \cos \left(n z_{2}\right) D_{1}^{2}$. Remark that $\left\{\frac{1}{2} \cos \left(n z_{2}\right)\right\}_{n \geq 1}$ is a sequence of $C^{\infty}$ functions, which converges strongly in $H^{-1}(Z)$ but not in $L^{2}(Z)$ (recall that by the Riemann-Lebesque lemma $\frac{1}{2} \cos \left(n z_{2}\right) \stackrel{w}{\rightarrow}$ 0 in $L^{2}(Z)$ and since $L^{2}(Z)$ embeds compactly in $H^{-1}(Z)$, we have $\frac{1}{2} \cos \left(n z_{2}\right) \stackrel{s}{\rightarrow} 0$ in $H^{-1}(Z)$ ). Then $B_{n} \stackrel{G}{\rightarrow} B=-\Delta$ and so Theorem 2 is applicable for systems monitored by parabolic partial differential equations involving these operators. In particular then we have convergence of the corresponding optimal values.

If $N=1$, the situation is simpler. In this case the partial differential operator is $-\frac{d}{d z}\left(a(z, \lambda(z)) \frac{d x}{d z}\right.$ and hypothesis $H(a)_{1}$ takes the following form:

$\underline{H(a)_{2}}$ for every $\lambda \in \Lambda$ and almost all $z \in Z, m_{1} \leq a\left(z, \lambda(z) \leq m_{2}\right.$ and if $\lambda \rightarrow \lambda$ in $\Lambda$ then $\frac{1}{a\left(\cdot, \lambda_{n}(\cdot)\right)} \stackrel{w^{*}}{\rightarrow} \frac{1}{a(\cdot, \lambda(\cdot))}$ in $L^{\infty}(Z)$.

Under this hypothesis, we know (cf. Dal Maso [3]), that

$$
\Gamma_{\text {seq }}\left(\mathbb{N}, w-H_{0}^{1}(Z)\right) \Phi\left(x, Z^{\prime}, \lambda_{n}\right)=\Phi\left(x, Z^{\prime}, \lambda\right),
$$

where $\Phi: H_{0}^{1}(Z) \times \mathscr{U} \times \Lambda \rightarrow \mathbb{R}$ is given by $\Phi\left(x, Z^{\prime}, \lambda^{\prime}\right)=\int_{Z^{\prime}} a\left(z, \lambda^{\prime}(z)\right)\left(\frac{d x}{d z}\right)^{2} d z$. So again Proposition 2 is valid and so we state the following result.

THEOREM 3. If hypotheses $H(a)_{2}, H(f), H(\theta)$ and $H(L)$ hold, then for all $\lambda \in \Lambda$, $Q(\lambda) \neq \emptyset$, the set-valued map $\lambda \rightarrow Q(\lambda)$ is u.s.c. from $\Lambda$ into the nonempty, weakly contact subsets of $W(T) \times L^{2}(T, H)$ and $\lambda \rightarrow m(\lambda)$ is continuous.

This is the case for example if $a_{n}(z)=1+e^{n z}$ and $a_{n}(z)=1$. Then $\frac{1}{a_{n}} \stackrel{w^{*}}{\rightarrow} \frac{1}{a}=1$ in $L^{\infty}(Z)$. Note that $\left\|a_{n}-a\right\|_{\infty}=1$. So $a_{n} \nrightarrow$ a strongly in $L^{\infty}(Z)$.

REMARK 4. This type of coefficient convergence was considered by Sokolowski [10]. 
Finally, we mention that the framework of this paper allows us also to treat optimal control problems with homogenization in the dynamics. In this case in the context of semilinear systems $a_{i j}^{n}(z)=a_{i j}\left(\gamma_{n} z\right)$ with $a_{i j}$ periodic and $\gamma_{n} \rightarrow \infty$ (see Dal Maso [3], Chapter 24). Also we can investigate systems with controls in the coefficients (see Sokolowski [10]).

\section{Acknowledgement}

The author wishes to thank the two anonymous referees for their corrections and constructive criticisms.

\section{References}

[1] G. Buttazzo, 'Semicontinuiuty, relaxation and integral representation in the calculus of variations Pitman Research Notes in Math. Series 207 (Longman Scientific and Technical, Essex, United Kingdom, 1989).

[2] G. Buttazzo and G. Dal Maso, " $\Gamma$-convergence and optimal control problems", J. Optim. Theory Appl. 38 (1982) 385-407:

[3] G. Dal Maso, An Introduction to $\Gamma$-convergence (Birkhäuser, Boston, 1993).

[4] F. S. DeBlasi and J. Myjak, "On continuous approximations for multifunctions", Pacific J. Math. 123 (1986) 9-31.

[5] A. Defranceschi, " $\Gamma$-convergence of cyclically monotone operators", Aymptotic Anal. 2 (1989) 21-37.

[6] A. G. Kolpakov, "Г-convergence of a class of evolution operators", Siberian Math. J. 29 (1988) 233-244.

[7] J.-L. Lions, Optimal control of systems governed by partial differential equations (Springer, New York, 1971).

[8] N. S. Papageorgiou, "On the set of solutions of a class of nonlinear evoluiton inclusions", Kodai Math. J. 15 (1992) 387-402.

[9] J. Simon, "Compact sets in the space $L^{p}(0, T ; B)$ ", Annali di Matematica Pura ed Applicata CLII (1987) 65-96.

[10] J. Sokolowski, "Optimal control in coefficients for weak variational problems in Hilbert space", Appl. Math. Optim. 7 (1981) 283-293.

[11] S. Spagnolo, "Sulla convergenza di soluzioni di equazioni paraboliche ed ellittiche", Ann. Scuola Normale Sup. Pisa 22 (1968) 571-597.

[12] D. Wagner, "Survey of measurable selection theorems", SIAM J. Control Optim. 15 (1977) 859903.

[13] E. Zeidler, Nonlinear functional analysis and its applications II (Springer, New York, 1990).

[14] V. Zhikov, S. Kozlov and O. Oleinik, " $\Gamma$-convergence of parabolic operators", Russian Math. Surveys 36 (1981) 9-60. 